\title{
The maximum standardized uptake values on positron emission tomography of a non-small cell lung cancer predict stage, recurrence, and survival
}

Robert James Cerfolio, MD, FACS, FCCP, ${ }^{\text {a }}$ Ayesha S. Bryant, MSPH, ${ }^{\mathrm{b}}$ Buddhiwardhan Ohja, MD, MPH, ${ }^{\mathrm{c}}$ and Alfred A. Bartolucci, $\mathrm{PhD}^{\mathrm{d}}$

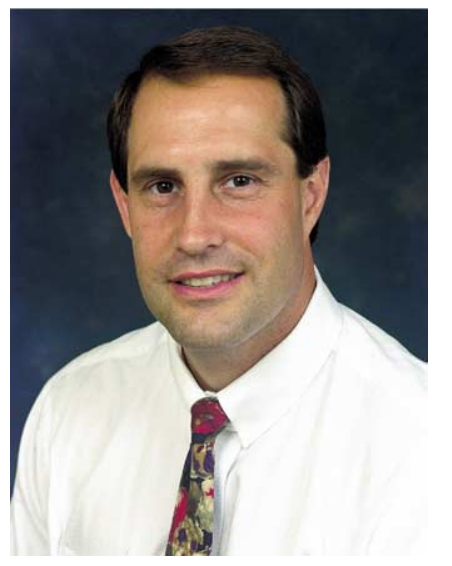

Dr. Cerfolio
Objective: We sought to assess whether the standard uptake value of a pulmonary nodule is an independent predictor of biologic aggressiveness.

Methods: This is a retrospective review of a prospective database of patients with non-small cell lung cancer. Patients had dedicated positron emission tomography scanning with F-18 fluorodeoxyglucose, with the maximum standard uptake value measured. All suspicious nodal and systemic locations on computed tomographic and positron emission tomographic scanning underwent biopsy, and when indicated, resection with complete lymphadenectomy was performed.

Results: There were 315 patients. Multivariate analysis showed patients with a high maximum standard uptake value $(\geq 10)$ were more likely to have poorly differentiated tumors (risk ratio, $1.5 ; P=.005$ ) and advanced stage (risk ratio, 1.9; $P=$ .010 ) and were less likely to have their disease completely resected (risk ratio, 3.7; $P=.004)$. Maximum standard uptake value was the best predictor of disease-free survival (hazard ratio, 2.5; $P=.039$ ) and survival (hazard ratio, 2.8; $P=.001$ ). Stage-specific analysis showed that patients with stage IB and stage II disease with a maximum standard uptake value of greater than the median for their respective stages had a lower disease-free survival at 4 years $(P=.005$ and .044$)$. The actual 4-year survival for patients with stage Ib non-small cell lung cancer was $80 \%$ versus $66 \%(P=.048)$, for stage II disease it was $64 \%$ versus $32 \%(P=.028)$, and for stage IIIa disease it was $64 \%$ versus $16 \%(P=.012)$ for the low and high maximum standard uptake value groups, respectively.

From the Section of Thoracic Surgery, University of Alabama at Birmingham, and the Division of Cardio-Thoracic Surgery, Department of Surgery, ${ }^{a}$ Birmingham Veterans Administration Hospital, Birmingham, Ala; the Department of Epidemiology $y^{\mathrm{b}}$ and the Department of Biostatistics, ${ }^{\mathrm{d}}$ School of Public Health, University of Alabama at Birmingham, Birmingham, Ala; and the Department of Nuclear Medicine, ${ }^{\mathrm{c}}$ University of Alabama at Birmingham, Birmingham, Ala.

Received for publication July 15,2004 ; revisions received Nov 1, 2004; accepted for publication Nov 9, 2004.

Address for reprints: Robert J. Cerfolio, MD, Associate Professor of Surgery, Chief of Thoracic Surgery, Division of Cardiothoracic Surgery, University of Alabama at Birmingham, 1900 University Blvd, THT 712, Birmingham, AL 35294 (E-mail: Robert.cerfolio@ccc.uab.edu).

J Thorac Cardiovasc Surg 2005;130:151-9

$0022-5223 / \$ 30.00$

Copyright () 2005 by The American Association for Thoracic Surgery

doi:10.1016/j.jtcvs.2004.11.007
Conclusions: The maximum standard uptake value of a non-small cell lung cancer nodule on dedicated positron emission tomography is an independent predictor of stage and tumor characteristics. It is a more powerful independent predictor than the TNM stage for recurrence and survival for patients with early-stage resected cancer. This information might help guide treatment strategies.

$\mathrm{T}$ The treatment of non-small cell lung cancer (NSCLC) depends on the stage. ${ }^{1}$ Therefore the clinician spends significant time, effort, and money performing tests to assess the stage. Yet even after careful clinical staging followed by complete resection, the 5-year survival for patients with pathologic stage Ia, Ib, II, and IIIa disease is only $67 \%, 57 \%, 47 \%$, and $23 \%$, respectively. ${ }^{1}$ Integrated positron emission tomography (PET)-computed tomography (CT) with F-18 fluorodeoxyglucose (FDG; FDG-PET/CT) or dedicated PET (FDG-PET) is an increasingly available noninvasive test that has been shown to be useful for the evaluation of an indeterminate pulmonary nodule, the staging of mediastinal lymph nodes, the evaluation of local nodal and distant metastases, and the response to chemoradiotherapy. ${ }^{2-8}$ In addition, there might be another not yet fully explored role. FDG-PET measures the standardized uptake value (SUV) of a pulmonary mass, which quan- 
TABLE 1. Patient characteristics

\begin{tabular}{lc}
\hline & $\begin{array}{c}\text { Overall, } \\
\mathbf{n}=\mathbf{3 1 5}\end{array}$ \\
\hline Sex & \\
Male & $178(57 \%)$ \\
Female & $137(43 \%)$ \\
Median age, y (range) & $66(25-88)$ \\
Surgery type* & \\
EUS-FNA biopsy of N2 nodes & 14 \\
Mediastinoscopy & 67 \\
Segmentectomy & 10 \\
Video-assisted thoracoscopic wedge resection & 12 \\
$\quad$ of M1, met nodules & 10 \\
Wedge & 210 \\
Lobectomy & 5 \\
Pneumonectomy & \\
\hline
\end{tabular}

EUS-FNA, Endoscopic ultrasound fine-needle aspirate. *Excludes $17 \mathrm{pa}-$ tients with stage IV cancer of brain or bone who did not undergo surgical intervention and patients who might have had more than one procedure.

tifies the glucose avidity of the tumor. This value is calculated by the software contained within the PET machine and is thus relatively consistent from one PET center to another. The objective of this study was to assess whether the maximum SUV, which is less variable than the mean SUV, ${ }^{9}$ represents a tumor's in vivo virulence or a quantification of its biology.

\section{Methods \\ Patients}

This is a retrospective review of a prospective database. Patients who presented to one surgeon (R.J.C.) between January 2001 and June 2004 with an indeterminate pulmonary nodule or a biopsyproved NSCLC who underwent FDG-PET scanning were eligible to participate in this study. Patients were excluded if they were less than 19 years old, had a history of type I diabetes, had any chemotherapy or radiotherapy before the maximum SUV calculation on PET, or had histology that was not NSCLC. All patients were staged and included in the maximum SUV as a predictor of stage analysis of this study, as well as the survival analysis. However, only those patients who underwent complete (R0) resection were included in the disease-free survival analysis of this study.

\section{Imaging}

FDG-PET scans were performed on a dedicated ECAT EXACT PET scanner (CTI, Knoxville, Tenn) or on an integrated PET-CT scanner (GE Discovery LS PET-CT Scanner, Milwaukee, Wis). Patients were asked to fast for 4 hours and then subsequently received $555 \mathrm{MBq}(15 \mathrm{mCi})$ of FDG intravenously followed by PET after 1 hour. The scans were performed from the skull base to the midthigh level. Attenuation correction of PET images for the ECAT system was performed with standard transmission scanning by using 68 Germanium sources (3 rods). The scanning time for emission PET was 6 minutes, and transmission with 68 Germa- nium rods was 4 minutes per bed position. For the Discovery system, a CT examination was used for attenuation correction of PET images. The scanning time for emission PET was 5 minutes per bed position. Iterative reconstruction with $\mathrm{CT}$ attenuation correction was performed. The most recent CT scan of the chest was available for visual correlation. Maximum SUV was determined by drawing regions of interest on the attenuation-corrected FDG-PET images around the primary tumor. It was then calculated by using the software contained within the PET or PET-CT scanner by using the following formula ${ }^{10}$ :

$$
\text { Maximum } S U V=C(\mu \mathrm{Ci} / m L) / I D(\mu \mathrm{Ci}) / w(k g),
$$

where $\mathrm{C}$ is defined as the activity at a pixel within the tissue defined by a region of interest, and ID is defined as the injected dose per kilogram of patient's body weight (w). The maximum SUV within the selected regions of interest was used throughout this study exclusively.

\section{Procedures, Staging, and Surgical Intervention}

Patients were meticulously staged. All suspicious N2, N3, or M1 areas (maximum SUV, >2.5) underwent biopsy before pulmonary resection. Mediastinoscopy was used for biopsy of suspicious lymph nodes in the paratracheal area (stations $2 \mathrm{R}, 4 \mathrm{R}, 2 \mathrm{~L}$, and $4 \mathrm{~L}$ ), and endoscopic transesophageal ultrasonography was used for biopsy of suspicious posterior aorta-pulmonary window nodes (6), subcarinal nodes (7), periesophageal nodes (8), and inferior pulmonary ligament nodes (9). ${ }^{11}$ Patients with suspected M1 disease in the liver, adrenal gland, or contralateral lung underwent definitive biopsy to prove or disprove M1 cancer. If the bone or brain was suspected to harbor metastases, magnetic resonance imaging was considered the standard reference. If patients had biopsy-proved N3 or M1 disease, the stage was recorded, but they were excluded from the disease-free analysis. If there was no evidence of $\mathrm{N} 2$ or higher disease, patients underwent thoracotomy, pulmonary resection, and complete thoracic lymphadenectomy. Pathologic review was performed by using standard techniques, and immunohistochemical staining was used when appropriate. The pathologic stage was assessed by the international staging system. ${ }^{11}$

Patients were followed for cancer recurrence and survival. Follow-up data were obtained every 3 months for the first 2 years and every 6 months afterward. A chest radiograph was performed every 3 months, and a chest CT with intravenous contrast was performed every 6 months. FDG-PET was also used at 6 months in selected patients. In addition, if patients became symptomatic, appropriate testing (ie, bone and brain scanning) was performed as well. Information was obtained from clinic letters, hospital computer information systems, treatment updates, letters from oncology clinics and other physicians, social security death indexes, and telephone calls. The University of Alabama at Birmingham's institutional review board approved both the prospective database used for this study and this trial.

\section{Statistics: Definitions}

The maximum SUV was evaluated statistically by 3 methods. The first method evaluated the SUV as a continuous value, irrespective of the patient's stage. The second method was the maximum SUV within each stage of NSCLC, termed the stage-specific maximum SUV. In this method, after patients were pathologically staged, they were divided into 1 of 2 groups. If they had a maximum SUV 
TABLE 2. Univariate analysis of patient population, tumor characteristics, and survival

\begin{tabular}{|c|c|c|c|c|c|c|c|}
\hline & $\begin{array}{l}\text { No. of } \\
\text { patients } \\
\text { (315) }\end{array}$ & $\begin{array}{l}\text { Median } \\
\text { maximum } \\
\text { SUV }\end{array}$ & $\begin{array}{c}P \text { value } \\
\text { (for maximum } \\
\text { SUV) }\end{array}$ & $\begin{array}{c}\text { Mean } \\
\text { survival (y) }\end{array}$ & $\begin{array}{c}P \text { value } \\
\text { (for survival) }\end{array}$ & $\begin{array}{c}\text { Mean } \\
\text { disease-free } \\
\text { survival }(y)\end{array}$ & $\begin{array}{c}P \text { value } \\
\text { (for disease-free } \\
\text { survival) }\end{array}$ \\
\hline \multicolumn{8}{|l|}{ Stage } \\
\hline la & 59 & 6.9 & & 3.1 & & 2.5 & \\
\hline $\mathrm{lb}$ & 82 & 10.3 & & 2.4 & & 2.3 & \\
\hline II & 57 & 12.9 & $<.001$ & 2.2 & $<.001$ & 2.0 & .010 \\
\hline Illa & 72 & 14.2 & & 1.6 & & - & \\
\hline IIlb & 15 & 12.7 & & 0.98 & & - & \\
\hline IV & 30 & 15.9 & & 1.3 & & - & \\
\hline \multicolumn{8}{|l|}{ Histology } \\
\hline Squamous cell & $162(54 \%)$ & 13.2 & .140 & 2.4 & .21 & 2.4 & .176 \\
\hline Adenocarcinoma & $97(33 \%)$ & 8.7 & & 2.7 & & 1.8 & \\
\hline Other NSCLC & $39(13 \%)$ & & & 2.6 & & 2.2 & \\
\hline \multicolumn{8}{|l|}{ Tumor differentiationt } \\
\hline Well & $42(18 \%)$ & 3.9 & $<.001$ & 2.4 & .01 & 2.2 & .840 \\
\hline Moderately & $108(46 \%)$ & 10.1 & & 1.5 & & 1.8 & \\
\hline Poorly & $87(34 \%)$ & 15.1 & & 0.75 & & 1.0 & \\
\hline \multicolumn{8}{|c|}{ Lymphovascular invasion* } \\
\hline No & $170(75 \%)$ & 7.6 & .0442 & 2.4 & .003 & 2.3 & .820 \\
\hline Yes & $57(25 \%)$ & 12.5 & & 1.8 & & 1.5 & \\
\hline \multicolumn{8}{|l|}{ FDG-PET type } \\
\hline Dedicated scanner & $75(24 \%)$ & 9.8 & .129 & 2.5 & .73 & 1.9 & .870 \\
\hline Integrated scanner & $240(76 \%)$ & 9.9 & & 2.6 & & 2.3 & \\
\hline \multicolumn{8}{|l|}{ Resection } \\
\hline Yes & $225(71 \%)$ & 9.4 & $<.001$ & 3.1 & $<.001$ & - & - \\
\hline No & $90(29 \%)$ & 15.2 & & 1.5 & & - & - \\
\hline \multicolumn{8}{|l|}{ Neoadjuvant treatment } \\
\hline Yes & $35(11 \%)$ & 11.7 & .331 & 3.1 & .088 & 0.91 & .010 \\
\hline No & $280(89 \%)$ & 11.0 & & 2.1 & & 2.3 & \\
\hline \multicolumn{8}{|l|}{ Adjuvant treatment } \\
\hline Yes & $23(7 \%)$ & 12.8 & .148 & 1.4 & .108 & 2.3 & .560 \\
\hline No & $292(93 \%)$ & 10.2 & & 2.8 & & 2.8 & \\
\hline \multicolumn{8}{|l|}{ SUV } \\
\hline$<10$ & $162(51 \%)$ & - & - & 3.2 & $<.001$ & 2.5 & .032 \\
\hline$\geq 10$ & $153(49 \%)$ & & & 1.6 & & 2.0 & \\
\hline
\end{tabular}

NSCLC, Non-small cell lung cancer; FDG-PET, F-18 fluorodeoxyglucose positron emission tomography; SUV, standardized uptake value. *Excludes 17 patients with stage IV cancer of brain or bone who had no tissue type available. †Pathologic characteristics were not reported for all patients. Patients who underwent mediastinoscopy, endoscopic ultrasound fine-needle aspirate biopsy, or who were not resected because of metastatic disease did not have degree of differentiation and lymphovascular invasion reported.

greater than or equal to the median maximum SUV for that stage, they were placed in the high group for that stage, and if the value was lower, they were placed in the low group. The outcomes of these 2 groups in the same stage were then compared. The third method used maximum SUV as a binary variable. A cutoff point was identified by the log-rank test and a generalized Wilcoxon test.

In the disease-free survival analysis, failure (or recurrence of NSCLC) was defined as biopsy-proved NSCLC. If the biopsies were performed at outside institutions, pathology reports were obtained. Disease-free survival was defined as patients who were alive without recurrence. Operative mortality was defined as a patient who died before hospital discharge or within 30 days of the operative procedure.

\section{Statistical Analyses}

The primary end point was survival, which was from the date of surgical intervention to the date of the last follow-up or death. Patients who were still alive at the end of our study were censored. Disease-free survival was measured only for those who underwent complete R0 resection. A univariate analysis of all variables was performed initially to assess for differences among the maximum SUV, survival, and disease-free survival for variables. Univariate analyses were performed with a 2-sided log-rank test. ${ }^{12} \mathrm{~A} \chi^{2}$ analysis was used for discrete variables, with a $P$ value of less than .05 according to the 2-tailed Fisher exact test used to select factors with potential significance. Analysis of variance was used for discreet nondichotomous variables. For continuous variables, the 
TABLE 3. Variables found to be significantly associated with a higher maximum standardized uptake value on multivariate analysis

\begin{tabular}{lccc}
\hline Variable & $\begin{array}{c}\text { Relative } \\
\text { risk }\end{array}$ & $\begin{array}{c}\mathbf{9 5 \%} \text { Confidence } \\
\text { interval }\end{array}$ & $\boldsymbol{P}$ value \\
\hline $\begin{array}{l}\text { Higher stage (III or IV) } \\
\begin{array}{l}\text { Differentiation (moderate- } \\
\text { poor) }\end{array}\end{array}$ & 1.9 & $1.4-3.5$ & .010 \\
\begin{tabular}{l} 
Not resected \\
\hline
\end{tabular} & 3.7 & $1.2-5.5$ & .005 \\
\end{tabular}

TABLE 4. The median value of the maximum standardized uptake value and mean survival time of the pulmonary nodule on the basis of the $T, N$, and $M$ status of the patient*

\begin{tabular}{lcc}
\hline TNM staging & $\begin{array}{c}\text { No. of } \\
\text { patients }\end{array}$ & $\begin{array}{c}\text { Median maximum } \\
\text { sUV }\end{array}$ \\
\hline T1 N0 M0 & 59 & 6.9 \\
T2 N0 M0 & 82 & 10.3 \\
T3 N0 M0 & 25 & 14.3 \\
T4 N0 M0 & 11 & 12.7 \\
N0 M0 & & \\
N1 M0 & 177 & 9.3 \\
N2 M0 & 35 & 12.2 \\
N3 M0 & 72 & 14.2 \\
M0 & 3 & 15.8 \\
M1 & 285 & 10.6 \\
\hline
\end{tabular}

SUV, Standardized uptake value. *Univariate analysis.

Student $t$ test or the Mann-Whitney $U$ test was used to compare means for nonnormally distributed variables. All comparisons were 2 sided. Variables with the potential for a significant difference between groups on the basis of the results of the univariate analyses were entered as candidate variables in a multivariate analysis with a Cox proportional-hazards model with both forward and backward stepwise inclusion of factors, with an inclusion criterion of a $P$ value of .05 or less. Survival analysis for differences among the high-SUV and low-SUV groups was performed by using the Kaplan-Meier method. All statistical analysis was performed with SAS v. 8.02 software (SAS Institute, Inc, Cary, NC).

\section{Results}

\section{Patient Characteristics}

There were 315 patients with NSCLC, and their characteristics are shown in Table 1. There were 7 (2.2\%) operative deaths, and 12 patients were lost to follow-up. The median follow-up in the remaining 296 patients was 26 months (range, 6-42 months). Two hundred twenty-five (71\%) patients underwent complete resection. Most patients with stage III and IV disease did not. However, some patients with unsuspected $\mathrm{N} 2$ disease underwent complete resection, and 35 patients with biopsy-proved N2 disease underwent neoadjuvant therapy and then (after confirmed downstaging) underwent complete resection. There were 10 patients with stage IV NSCLC who underwent resection. Eight of them had an unsuspected nodule with the same histology in a different lobe. Two patients had solitary brain metastases, and all of their disease was resected.

There were no associations demonstrated between maximum SUV and other potential predictor variables, such as age, sex, surgical type, or type of PET scan performed. Also, there were no associations between treatment strategy and high and low SUV within the different stages. Table 2 shows the pathologic characteristics of the tumor, as well as the median survival associated with each variable. It demonstrates that as the stage of NSCLC increases, so does the median maximum SUV. It also shows that patients with squamous cell carcinoma had a higher maximum SUV than those with other types of NSCLC.

Table 3 represents the variables found to be significantly associated with a higher maximum SUV on multivariate analysis. Poorly differentiated tumors (risk ratio [RR], 1.5; $P=.005$ ), advanced stage (RR, $1.9 ; P=.010$ ), and tumors not completely resected (RR, $3.7 ; P=.004)$ were more likely to be associated with a higher maximum SUV.

\section{Maximum SUV as Predictor of Stage}

Table 4 shows the median maximum SUV of the pulmonary nodule on the basis of the T, N, and M status. The top part of the table demonstrates that as the $\mathrm{T}$ status increases in patients who are node negative, the maximum SUV of the primary NSCLC pulmonary nodule also increases. Interestingly, this progression is not noted for $\mathrm{T} 3$ to $\mathrm{T} 4$. The middle part of Table 4 shows that the maximum SUV increases as patients go from $\mathrm{N} 0$ to $\mathrm{N} 3$, and the bottom third shows the same as the patient goes from M0 to M1.

\section{Maximum SUV as a Predictor of Recurrence}

The 4-year disease-free survival rates for patients who underwent completely resection were calculated, comparing those with high maximum SUV with those with a low maximum SUV within each stage. Figure $1, A$, shows this for patients with stage Ib NSCLC (all pathologically staged T2 N0 M0). The disease-free survival was $92 \%$ for the low maximum SUV group and 51\% for the high maximum SUV group $(P=.005)$. Figure $1, B$, shows this for patients with stage II disease. The disease-free survival for patients with stage II NSCLC was 64\% for the low maximum SUV group and $47 \%$ for the high maximum SUV group $(P=.044)$. Although it was not significant for patients with stage Ia NSCLC (curves not shown), it was $100 \%$ versus $70 \%$, respectively.

Variables found to correlate with disease-free survival according to the univariate analysis are listed in Table 2 . Patients with a shorter disease-free survival were more 

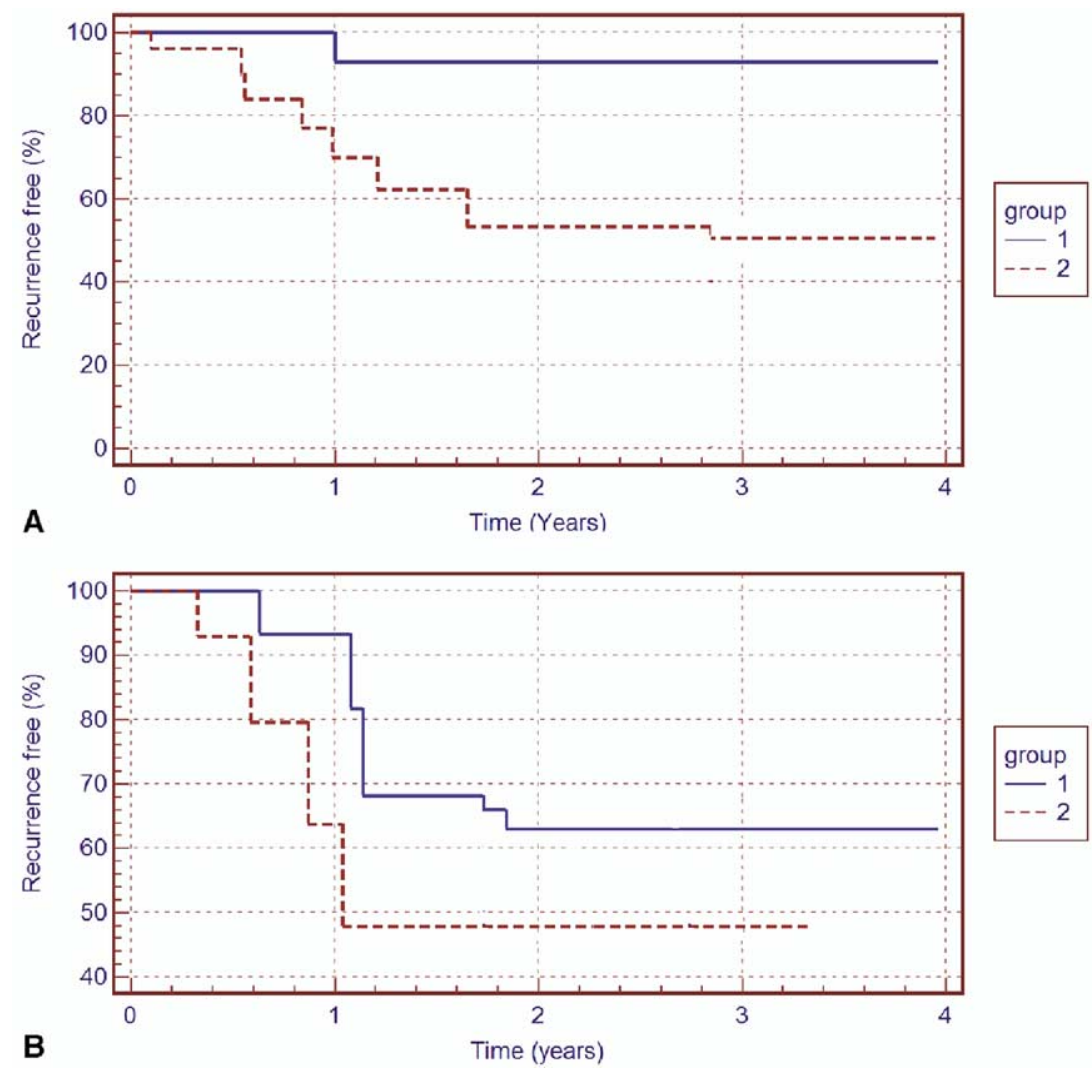

Figure 1. Kaplan-Meier curves depicting disease-free survival of NSCLC comparing patients with a low maximum SUV with those with a high maximum SUV by stage. Group 1, Patients with a maximum SUV lower than the median maximum SUV in that stage (low maximum SUV group). Group 2, Patients with a maximum SUV greater than or equal to the median maximum SUV in that stage (high maximum SUV group). A, Stage lb NSCLC $(P=.005)$; $B$, stage II NSCLC (P = .044).

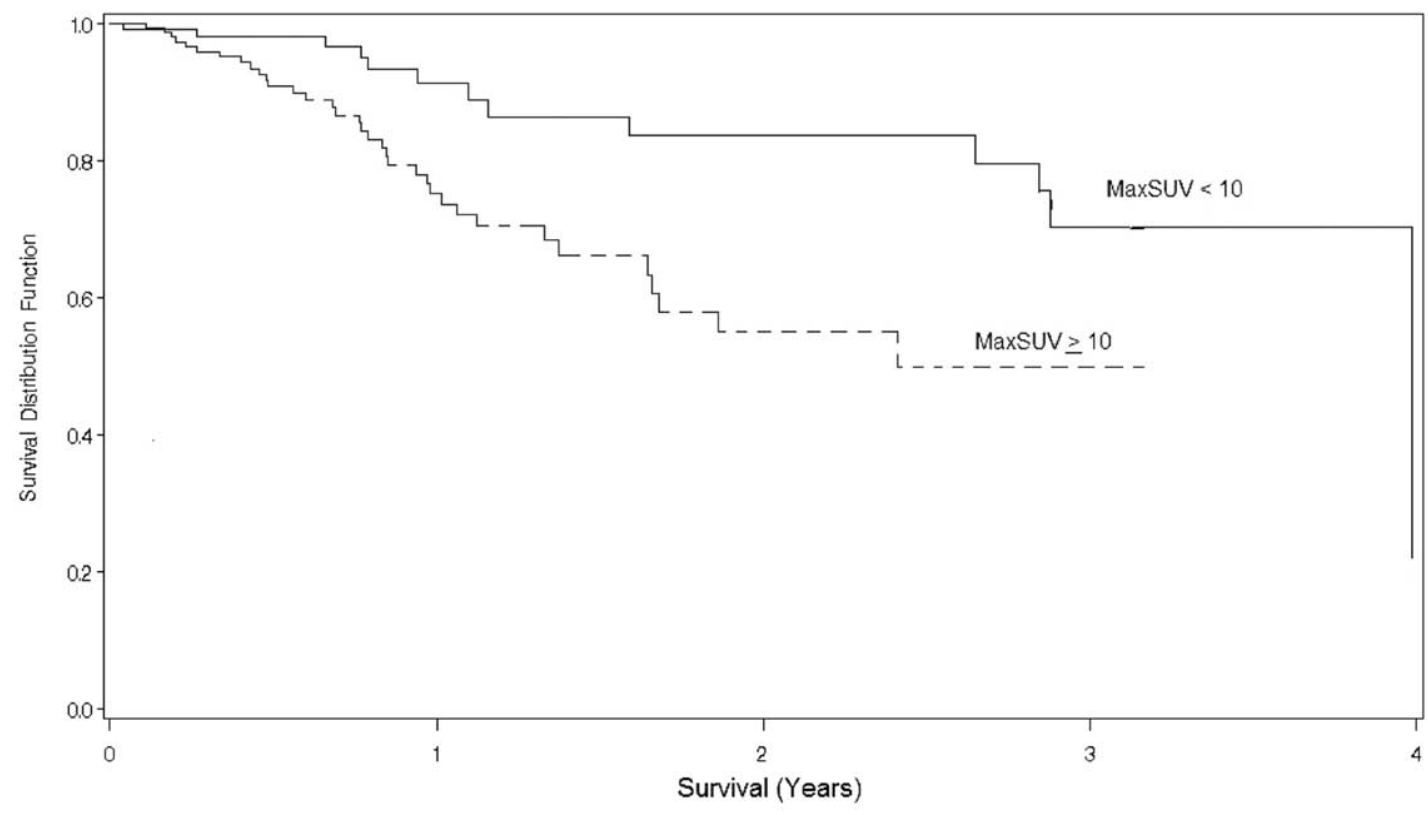

Figure 2. Cox proportional hazard survival curve for all 315 patients stratified by maximum SUV. 

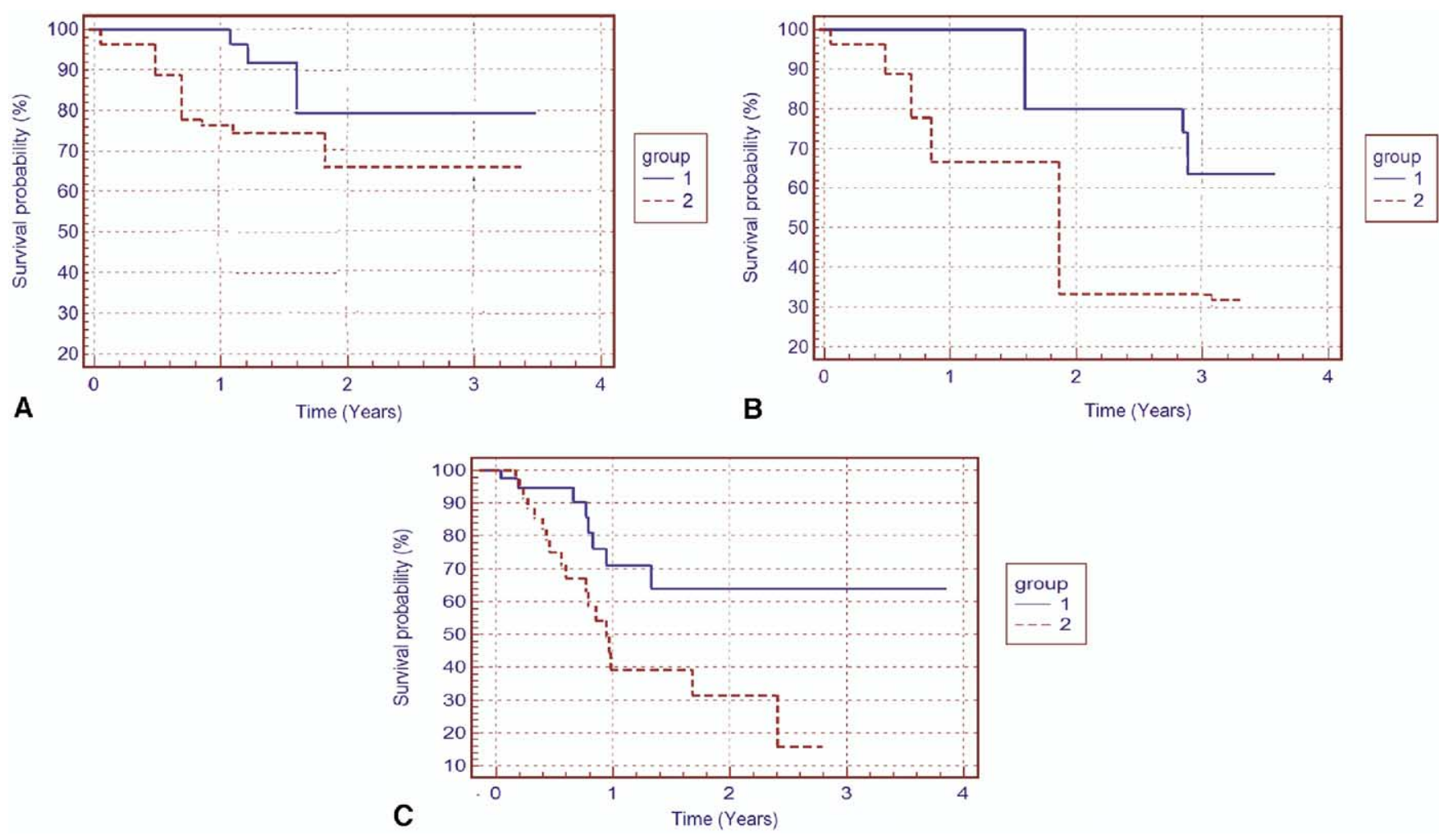

Figure 3. Kaplan-Meier curves depicting the actual survival for patients with a low maximum SUV compared with those with a high maximum SUV stratified by stage. Group 1, Patients with a maximum SUV lower than the median maximum SUV in that stage (low maximum SUV group). Group 2, Patients with a maximum SUV greater than or equal to the median maximum SUV in that stage (high maximum SUV group). A, Stage lb NSCLC (P=.048); B, stage II NSCLC $(P=.028) ;$ C, stage IIIA $(P=.0120)$.

likely to have had higher stage and a higher maximum SUV of 10 or greater. Of these candidate variables, only an SUV of greater than 10 was a predictor of a shorter disease-free (RR, 2.5; $P=.039)$ survival.

\section{Maximum SUV as a Predictor of Survival}

Figure 2 shows the Kaplan-Meier survival curves for all patients in this study irrespective of stage or treatment stratified by maximum SUV of greater than or equal to 10 and less than 10. The mean survival for the group with a maximum SUV of less than 10 was 3.2 years, whereas it was 1.6 years for those with a maximum SUV of 10 or greater $(P<.001)$.

Figure 3 shows the survival curves for those patients with stage Ib, II, and IIIa NSCLC stratified by stage and by the median maximum SUV within each stage. Patients with low maximum SUV for stage Ib, II, and IIIa disease all had a statistically significant greater 4-year survival rate than those within the high maximum SUV group: $80 \%$ versus $66 \%(P=.048)$ for stage $\mathrm{Ib}$ disease, $64 \%$ versus $32 \%(P=$ $.028)$ for stage II disease, and $64 \%$ versus $16 \%(P=.012)$ for stage IIIa disease. Although it was not statistically significant for predicting survival for patients with stage Ia disease, the 3 patients who died all were in the maximum SUV group. Table 5 shows the multivariable analysis for survival. We found that the maximum SUV was the strongest predictor of survival (hazard ratio, 2.8; $P<.001$ ).

TABLE 5. Variables found to be significantly associated with survival, as determined by using the multivariable Cox proportional hazard regression

\begin{tabular}{lccc}
\hline Variable & $\begin{array}{c}\text { Relative } \\
\text { risk }\end{array}$ & $\begin{array}{c}\text { 95\% Confidence } \\
\text { interval }\end{array}$ & $\boldsymbol{P}$ value \\
\hline Stage (I/II vs III/IV) & 2.15 & $1.2-5.9$ & .032 \\
Tumor differentiation (well & 0.81 & $0.301-2.195$ & .234 \\
$\quad$ vs moderate-poorly) & & & \\
Lymphovascular invasion & 0.45 & $0.85-2.36$ & .345 \\
SUV ( $\geq 10$ vs $<10)$ & 2.80 & $2.1-6.4$ & .001 \\
Resection & 1.53 & $1.1-2.9$ & .049 \\
\hline
\end{tabular}

SUV, Standardized uptake value. 
In addition, we separately compared the survivals of 3 groups of patients. The first group was those who underwent complete (R0) resection only and had no other therapy. The second group was those who had R0 resection but had unsuspected $\mathrm{N} 2$ disease on final pathology and had adjuvant carboplatinum-based chemotherapy. The third group was patients who had biopsy-proved $\mathrm{N} 2$ disease preoperatively, received neoadjuvant therapy with 4500 to $6000 \mathrm{cGy}$ and carboplatinum-based chemotherapy, and then underwent R0 resection. Although a trend was seen favoring those with a low maximum SUV, we found a statistically significant difference only in the first group $(P<.001)$. Patients who had an R0 resection only who had a maximum SUV of 10 or greater had a worse survival than those with a maximum SUV of less than 10.

\section{Discussion}

We found that maximum SUV on a dedicated PET scan is a powerful independent predictor of some of the pathologic characteristic of NSCLC and of recurrence patterns and survival in patients with early resected disease. In fact, it was a better predictor of survival than the current TNM staging classification. The clinical importance of this study depends on one's current belief of the best treatment of lung cancer. Some studies have shown that preoperative chemotherapy before resection in patients with stage Ib and II NSCLC $^{13}$ and with stage IIIa disease might increase survival.Very recently, the pendulum has begun to swing toward adjuvant therapy after a study reported in the New England Journal of Medicine ${ }^{18}$ and 2 oral presentations concerning 2 separate multi-institutional trials presented at the American Society of Clinical Oncology meeting in June 2004. All 3 of these reports suggest a survival benefit for adjuvant therapy. Yet despite all these data, the identification of patients who benefit the most from neoadjuvant therapy, adjuvant therapy, or both still remains unknown. If one believes that preoperative stage is now irrelevant and that all patients should receive adjuvant therapy, it is widely known that many patients are unable to complete this therapy. If one was able to identify patients who are at high risk of recurrence after resection, one might be more likely to push that patient toward completion. However, if one considers neoadjuvant therapy to be better than adjuvant therapy, the failure of clinical stage to predict pathologic stage is well known. Maximum SUV might be a better guide than the proposed clinical stage. Finally, if one believes that oncogenes, tumor serum markers, and protein expression that portends a worse survival ${ }^{19-23}$ are the best markers, this type of risk stratification information is often not known before resection (unlike the maximum SUV on a PET scan) and thus cannot guide the decision for neoadjuvant therapy. Even if it could be determined from a preoperative needle biopsy, few centers currently evaluate it. Thus we believe that these data presented above have significant clinical importance.

Our report has shown that the maximum SUV of NSCLC is positively correlated to the $\mathrm{T}$ status, $\mathrm{N}$ status, and $\mathrm{M}$ status and is an independent predictor of stage. It also independently predicts the likelihood of lymphovascular invasion, which might be a critical element for metastatic disease. This finding is consistent with pathophysiology. For example, Glut 1, a critical cellular component of glucose transfer that is overexpressed in patients with NSCLC, has been associated with a worse prognosis. ${ }^{24}$ This might explain the increase in glucose avidity and thus the high maximum SUV in patients with aggressive tumors. Some of these findings have been corroborated in previous studies (Ahuja and colleagues ${ }^{25}$ in 1998, Vansteenkiste and associates $^{26}$ in 1999, Dhital and coworkers ${ }^{4}$ in 2000, Jeong and colleagues $^{27}$ in 2002, Higashi and associates ${ }^{28}$ in 2002, Sasaki and coworkers ${ }^{29}$ in 2003, and Downey and colleagues ${ }^{30}$ in 2004), some of which are summarized in Table 6. Our study has also shown that maximum SUV is actually a better predictor of survival and disease-free recurrence than the current TNM staging system. We found the best value for the cutoff point of the maximum SUV to be 10 . The cutoff values found in the other articles cited above were as follows: 5 from Sasaki and coworkers ${ }^{29} 7$ from Jeong and colleagues, ${ }^{27}$ and 10 from Downey and colleagues. ${ }^{30}$

In our study a stage-specific analysis found that patients with the same disease stage who had complete resection but who had a higher maximum SUV were more likely to have cancer recurrence (significant for stages Ib and II) and shorter survival (significant for stage Ib, II, and IIIa disease) compared with those with low maximum SUV. We also found, as did Downey, that the survival for those patients who had complete resection and had no other therapy was worse if the maximum SUV was 10 or greater.

In fact, the maximum SUV might be not only a supplement to the current TNM staging system but also might aid in filling in some of the shortcomings of the current system. For example, patients with $\mathrm{T} 4$ and N3 disease are all labeled as having stage IIIb disease. Yet the outcomes vary for patients who have $\mathrm{T} 4$ disease from a malignant pleural effusion, T4 disease from having 2 nodules of the same type in the same lobe, and N3 disease. The current TNM classification system does not demonstrate the differences in recurrence and survival, but in this study the maximum SUV did. Of the 15 patients that had stage IIIb disease, 3 had N3 disease, and their median maximum SUV was 19.1, but the 4 patients who had T4 disease because of 2 cancers of the same histology in the same lobe had a median maximum SUV of only 5.1. In our practice we now choose to report a patient's clinical or pathologic stage as, for example, T2(11.2) N1 M0, with the maximum SUV provided in parentheses after the $\mathrm{T}$ status. 
TABLE 6. Recent studies evaluating the correlation between standardized uptake value, stage, and/or survival

\begin{tabular}{|c|c|c|c|}
\hline & No. & Time frame & Main findings \\
\hline Downey $(2004)^{30}$ & 100 & $5 y$ & $\begin{array}{l}\text { 1. Only evaluated surgically resected patients: maximum SUV }>10 \text {, worse survival } \\
\text { 2. Maximum SUV squamous carcinoma }>\text { adenocarcinoma. }\end{array}$ \\
\hline Sasaki $(2003)^{29}$ & 262 & $2 y$ & $\begin{array}{l}\text { 1. High maximum SUV }>5.0 \text {, worse } 1-y \text { disease-free survival and distant } \\
\text { metastases-free survival. }\end{array}$ \\
\hline Higashi $(2002)^{28}$ & 57 & $6.5 \mathrm{y}$ & $\begin{array}{l}\text { 1. SUV }>5 \text {, worse disease-free survival. } \\
\text { 2. SUV better predictor of recurrent disease than stage. } \\
\text { 3. Stage I with SUV low } 5 \text { did better. }\end{array}$ \\
\hline Vansteenkiste $(1999)^{26}$ & 125 & - & $\begin{array}{l}\text { 1. SUV }>7 \text {, worse survival. } \\
\text { 2. Tumor }<3 \mathrm{~cm} \text {, maximum SUV }<7 \text { did better. }\end{array}$ \\
\hline Ahuja $(1998)^{25}$ & 155 & 4 & 1. Mean SUV $>10$, worse survival. \\
\hline
\end{tabular}

SUV, Standardized uptake value.

These findings, if further corroborated by other centers, raise several questions that are best and maybe only truly answered with multi-institutional prospective randomized trials. For instance, does a patient with a completely resected stage Ia, Ib, or II NSCLC who has a high maximum SUV benefit more from adjuvant therapy than one with a low maximum SUV? Is such a patient one who might also benefit from neoadjuvant therapy? Should the maximum SUV, instead of just the clinical stage, be part of the equation that aids in that decision? Should a patient with a high maximum SUV but with an early clinically staged tumor receive other staging tests before resection (eg, brain magnetic resonance imaging or mediastinoscopy) to ensure his or her clinical stage is correct? Will he or she benefit from closer postoperative surveillance? Can tumor or gene markers be correlated with a high SUV and, if so, which ones? Can one identify an absolute number for the maximum SUV that is consistent across centers that is a marker for poor survival, or should it be stage specific?

In this report we chose to use the maximum SUV instead of the mean SUV because it is less variable, ${ }^{9}$ less subjective, and more reproducible and provides more information about the aggressiveness of a tumor. It does not change despite changes in technique, time between injection and scanning, or the individual reading the PET scan. For this study to have universal applicability, the patient's maximum SUVs should be the same or very similar at different PET centers. Most dedicated PET and integrated PET-CT scanners and all new machines have software packages that automatically calculate the maximum SUV, accounting for the various techniques used. Thus, the divergence of maximum SUV across centers should be small and will be even less problematic over time.

In conclusion, this is the largest report to show that the maximum SUV of a pulmonary nodule on FDG-PET scanning is an independent predictor of an NSCLC's biologic aggressiveness or its in vivo virulence. The maximum SUV independently predicts an NSCLC's likelihood of metastasizing to regional, hilar, and mediastinal lymph nodes, as well as to distant metastatic sites. It also predicts its propensity for lymphovascular invasion and recurrence rates. It more accurately predicts recurrence rates for stage Ib and II NSCLC and survival for patients with stage Ib, II, or IIIa NSCLC than the currently used TNM staging system. These 
provocative data should be verified by other centers, and then prospective randomized trials are required before one can fully assess the effect that maximum SUV will have on treatment strategies for patients with NSCLC. Although this article and its conclusions must obviously be confined to patients with NSCLC, some of its findings might be applicable to patients with other types of solid-organ cancers that feature squamous cell carcinoma or adenocarcinoma.

\section{References}

1. Spira A, Ettinger DS. Multidisciplinary management of lung cancer. N Engl J Med. 2004;350:379-92.

2. Cerfolio RJ, Buddhiwardhan O, Bryant AS, et al. The role of FGDPET scan in staging patients with non small cell carcinoma. Ann Thorac Surg. 2003;76:861-6.

3. Cerfolio RJ, Bryant AS, Winokur TS, et al. Repeat FDG-PET after neoadjuvant therapy is a predictor of pathologic response in patients with non-small cell lung cancer. Ann Thorac Surg. 2004;78:1903-9.

4. Dhital K, Saunders CA, Seed PT, O'Doherty MJ, Dussek J. (18)FFluorodeoxyglucose positron emission tomography and its prognostic value in lung cancer. Eur J Cardiothorac Surg. 2000;18:425-8.

5. McCain, TW, Dunagan DP, Chin R, et al. The usefulness of positron emission tomography in evaluating patients for pulmonary malignancies. Chest. 2000;118:1610-5.

6. Gupta, NC, Graeber GM, Bishop HA, et al. Comparative efficacy of positron emission tomography with fluorodeoxyglucose in evaluation of small, intermediate and large lymph node lesions. Chest. 2000;117: 773-8.

7. Pieterman RM, van Putten JWG, Meuzelaar JJ, et al. Preoperative staging of non-small cell lung cancer with positron emission tomography. $N$ Engl J Med. 2000;343:254-61.

8. Strauss LG. Sensitivity and specificity of positron emission tomography for the diagnosis of lymph node metasases. Recent Results Cancer Res. 2000;157:12-9.

9. Lee JR, Madsen MT, Bushnel D, et al. A threshold method to improve standardized uptake value reproducibility. Nucl Med Commun. 2000; 21:685-90.

10. Nabi HA, Zubeldia JM. Clinical applications of F18-FDG in oncology. J Nucl Med Technol. 2002;30:3-9.

11. Mountain CF. Revisions in the International Systems for Staging Lung Cancer. Chest. 1997;111:1710-7.

12. Cox DR. Regression models and life-tables. J R Stat Soc [B]. 1972; 34:187-220.

13. Depierre A, Milleron B, Moro-Sibilot D, et al. Preoperative chemotherapy followed by surgery compared with primary surgery in resectable Stage 1 (except T1N0), II and IIIa non-small cell lung cancer. J Clin Oncol. 1999;20:247-53.

14. Rosell R, Gomez-Codina J, Camps C, et al. A randomized trial comparing preoperative chemotherapy plus surgery with surgery alone in patients with non-small-cell lung cancer. N Engl J Med. 1994;330: 153-8.
15. Roth JA, Fossella F, Komaki R, et al. A randomized trial comparing perioperative chemotherapy and surgery with surgery alone in resectable stage IIIA non-small-cell lung cancer. J Natl Cancer Inst. 1994; 86:673-80.

16. Roth JA, Atkinson EN, Fossella F, et al. Long-term follow-up of patients enrolled in a randomized trial comparing perioperative chemotherapy and surgery with surgery alone in resectable stage IIIA non-small-cell lung cancer. Lung Cancer. 1998;21:1-6.

17. Rosell R, Gomez-Codina J, Camps C, et al. Preresectional chemotherapy in stage IIIA non-small-cell lung cancer: a 7-year assessment of a randomized controlled trial. Lung Cancer. 1999;26:7-14.

18. The International Adjuvant Lung Cancer Trial Collaborative Group. Cisplatin-based adjuvant chemotherapy in patients with completely resected non-small-cell lung cancer. N Engl J Med. 2004;350:351-60.

19. D'Amico TA, Aloia TA, Moore MB, et al. Molecular biologic substaging of stage I lung cancer according to gender and histology. Ann Thorac Surg. 2000;69:882-6.

20. Harpole DH, Herndon JE II, Young WG, et al. Stage I non-small cell lung cancer: a multivariate analysis of treatment methods and patterns. Cancer. 1995;76:787-96.

21. Harpole DH, Herndon JE, Wolfe WG, et al. A prognostic model of recurrence and death in stage I non-small cell lung cancer utilizing presentation, histopathology, and oncoprotein expression. Cancer Res. 1995;55:51-6.

22. Strauss GM, Kwiatkowski DJ, Harpole DH, et al. Molecular and pathologic markers in stage I non-small cell carcinoma of the lung. J Clin Oncol. 1995;13:1265-79.

23. Kwiatkowski DJ, Harpole DH, Godleski J, et al. Molecular pathologic substaging in 244 stage I non-small cell lung cancer patients: clinical implications. J Clin Oncol. 1998;16:2468-77.

24. Younes M, Brown RW, Stephenson M, et al. Overexpression of Glut-1 and Glut3 in stage I nonsmall cell lung carcinoma is associated with poor survival. Cancer. 1997;80:1046-51.

25. Ahuja V, Coleman RE, Herndon J, Patz EF Jr. The prognostic significance of fluorodeoxyglucose positron emission tomography imaging for patients with nonsmall cell lung carcinoma. Cancer. 1998;83:918-24.

26. Vansteenkiste JF, Stroobants SG, Dupont PJ, et al. Prognostic importance of the standardized uptake value on (18)F-fluoro-2-deoxy-glucose-positron emission tomography scan in non-small-cell lung cancer: an analysis of 125 cases. Leuven Lung Cancer Group. J Clin Oncol. 1999;10:3201-6.

27. Jeong HJ, Min JJ, Park JM, et al. Determination of the prognostic value of fluorodeoxyglucose uptake by using positron emission tomography in patients with non-small cell lung cancer. Nucl Med Commun. 2002;23:865-70.

28. Higashi K, Ueda Y, Arisaka Y. 18F-FDG uptake as a biologic prognostic factor for recurrence in patients with surgically resected nonsmall cell lung cancer. J Nucl Med. 2002;43:39-45.

29. Sasaki R, Komaki R, Macapinlac H, et al. SUV by FDG-PET predicts outcomes of NSCLC. Int J Radiat Oncol Biol Phys. 2003;57(suppl 2):S166.

30. Downey RJ, Akhurst T, Gonen M, et al. Preoperative F-18 fluorodeoxyglucose-positron emission tomography maximal standardized uptake value predicts survival after lung cancer resection. J Clin Oncol. 2004;22:3255-60. 